\title{
Dynamic Stokes shift in solution: Effect of finite pump pulse duration
}

\author{
Yuri Georgievskii, Chao-Ping Hsu, and R. A. Marcus \\ Division of Chemistry and Chemical Engineering, Noyes Laboratory of Chemical Physics, \\ California Institute of Technology, Pasadena, California 91125
}

(Received 14 August 1997; accepted 27 January 1998)

\begin{abstract}
The time-evolution of the fluorescence spectrum of a dissolved chromophore excited by an ultrafast pump pulse is considered. The average value of the energy difference of the solute in its excited and ground states is used to describe the relaxation of the maximum of the transient fluorescence spectrum to its equilibrium value (dynamic Stokes shift, DSS). A simple formula for the normalized DSS is obtained which generalizes an earlier standard classical expression and includes the effect of a pump pulse of finite duration. As an example, dielectric dispersion data are used for a dipolar solute in water to estimate the quantum correction to the standard DSS expression. The correction is negligible when the frequency of the pump pulse is close to the maximum in the absorption spectrum, but a deviation from the standard formula can be expected for the pump pulse tuned to a far wing of the absorption band of the chromophore. An expression is given for this deviation. (C) 1998 American Institute of Physics. [S0021-9606(98)50817-3]
\end{abstract}

\section{INTRODUCTION}

The solvation dynamics of dipolar and ionic solutes in polar solvents has attracted considerable attention in recent years, e.g., Refs. 1-7. This interest reflects the importance of the solvent dynamics in many chemical and physical processes in the condensed phase. ${ }^{8-11}$ Transient nonlinear spectroscopy has been one main tool for tracing the ultrafast dynamics of solvation. ${ }^{12-14}$ The loss of coherence between the ground and excited electronic states, which is caused by the solute-solvent interaction, is closely related to solvation dynamics. The photon echo technique ${ }^{15-22}$ and pump-probe spectroscopy ${ }^{23-26}$ have both been used to separate the inhomogeneous (slow) broadening of the spectral line associated with a particular electronic transition from the homogeneous (fast) electronic dephasing. Several new experimental techniques have been developed to trace electronic dephasing with femtosecond resolution. ${ }^{27-31}$ In a different, but related approach the optical Kerr effect has been used to study subpicosecond dynamics of the solvent as a whole..$^{32-35}$

Most of the experimental data on solvation dynamics in polar solvents have been obtained using a time-resolved fluorescence method with upconversion. ${ }^{36-46}$ The energy difference in the ground and excited electronic states of the solute is manifested through $\nu(t)$, the frequency of the fluorescence spectral maximum of the molecule. Its timeevolution (dynamic Stokes shift, DSS) reflects the solvation dynamics of the electronically excited solute. At the current level of resolution a DSS experiment permits a scanning of the solvent dynamics on a time scale ranging from less than 100 femtoseconds for fast relaxing solvents like water ${ }^{2}$ up to nanoseconds for "slow" solvents. ${ }^{47}$ For strongly coupled systems the time-resolved fluorescence experiments ${ }^{36-47}$ have been more extensively compared with theory and with computer simulations than photon echo ${ }^{15-22,27-31}$ or optical Kerr effect measurements. ${ }^{32-35}$

To interpret the results of a DSS measurement it is usu- ally assumed that the photoexcitation, i.e., the transition forming the molecule in the excited electronic state, occurs much faster than any relaxation of the solvent and so the excited molecule would appear initially in a solvent which is in equilibrium with the ground state of the molecule. ${ }^{48}$ It is also usually assumed that the solvent dynamics that is related to the evolution of the transient fluorescence spectrum can be described classically and that quantum effects in the solvent dynamics can be neglected.

Recent ultrafast DSS measurements ${ }^{2,36-38}$ have shown that the considerable part of relaxation $(\geq 50 \%)$ in many solvents composed of small molecules occurs on a very short time scale ( $\leqslant 100 \mathrm{fs}$ ), which becomes comparable to the pump pulse duration. Under these conditions the validity of the assumption that the state of the solvent immediately after the excitation is the same as before is no longer strictly valid. In the present article an estimate is made of the effect of the finite pump pulse duration on this time-development of the Stokes shift and of the extent to which the quantum dynamics of the solvent can modify the usual classical result. A model is described in Sec. II, and the dynamic Stokes shift is calculated in Sec. III, initially for an instantaneous pulse (Sec. III A) and then for a pulse of finite duration (Sec. III B). A physical interpretation of the principal equations is given in Sec. IV, and a summary is given in Sec. V.

\section{THE MODEL}

For treating the solvent dynamics several approaches come to mind. In one of these linear response theory is used, as Ovchinnikov and Ovchinnikova $\mathrm{did}^{49}$ in their application of the quantum field theoretical method of Abrikosov et al. ${ }^{50}$ In a similar spirit, Mukamel and coworkers ${ }^{51-53}$ used a cumulant expansion, based on second-order perturbation theory to treat systems which in molecular terms have nonlinear interactions. Neither treatment uses a molecular harmonic oscillator model. An approach which is, at first glance, quite 
different from these in validity, is the use of a molecular oscillator model-the well known spin-boson Hamiltonian. ${ }^{54}$ It gives results which are formally the same ${ }^{55}$ as those obtained by the other two methods, when the number of oscillators $N$ allowed to become infinite. This agreement is not accidental. Rather, it bears some analogy to the representation of a nonperiodic function by a Fourier integral, when the latter is regarded as the limit as $N$ tends to $\infty$ of a Fourier series representation of the function. We have chosen to use this spin-boson Hamiltonian approach, and then allowing $N$ to tend to $\infty$, because of its simplicity, although either of the other two methods could have been used instead to obtain the key equations, Eqs. (47) and (48). We note that these equations do not, for the above reason, contain any properties specific to molecular harmonic oscillators.

In the harmonic oscillator approach (with finite $N$, which at the end is allowed to become infinite), the solvent Hamiltonian $H_{g}$ in the ground electronic state can be written as $^{54,56-58}$

$$
H_{g}=\sum_{j} \frac{1}{2}\left(P_{j}^{2}+\omega_{j}^{2} Q_{j}^{2}\right)+U_{g},
$$

where $\omega_{j}, Q_{j}$, and $P_{j}$ are the frequencies, coordinates, and momenta of the "normal modes," respectively (massweighted coordinates). When the resulting change of electronic state after an electronic transition leads only to shifts of the normal modes but not to changes in their frequency, the solvent Hamiltonian $H_{e}$ in the excited electronic state can be written as ${ }^{54,56-58}$

$$
H_{e}=\sum_{j} \frac{1}{2}\left[P_{j}^{2}+\omega_{j}^{2}\left(Q_{j}+\frac{c_{j}}{\omega_{j}^{2}}\right)^{2}\right]+U_{e}
$$

where the coefficients $c_{j}$ uniquely characterize the shifts of equilibrium positions of the normal modes. The difference of the minima of the potential energies $\Delta U=U_{e}-U_{g}$ in the excited and ground electronic states, respectively, coincides with the free energy difference for this harmonic oscillator model.

A comment is relevant here about the role of intramolecular solute modes. In most experiments relatively large molecules (mostly dye molecules) with many nuclear degrees of freedom are used as solute probes, ${ }^{5}$ molecules in which the equilibrium nuclear configuration in the excited electronic state is different from the one in the ground electronic state. Vibrational modes of such solutes, changes in the ring modes in aromatic systems, for example, contribute considerably to the static Stokes shift and must be included in any model. The harmonic approximation for intramolecular modes is often used for electron transfer and other nonadiabatic electronic transitions. ${ }^{57,58}$ One can then assume that the sum in Eqs. (1) and (2) is not only over the solvent modes but also over the intramolecular solute modes, neglecting any change in their frequencies as a result of the electronic transition.

To describe the solvent dynamics related to the spectroscopy of the solute it is now customary to treat the energy difference of the excited and ground electronic states as a collective coordinate ${ }^{51-53}$

$$
X=H_{e}-H_{g}=\sum_{j} c_{j} Q_{j}+\Delta U+\lambda,
$$

where the "solvent reorganization energy" $\lambda$ is given in the harmonic oscillator model by

$$
\lambda=\sum_{j} c_{j}^{2} / 2 \omega_{j}^{2}
$$

The coordinate $X$ corresponds to the optical frequency of the vertical electronic transition at any specified values of the nuclear coordinates of the solvent. It can be referred to as a generalized "solvation coordinate." A similar idea was used earlier in electron transfer theory. ${ }^{60-65}$ Statistical and temporal properties of the solvation coordinate are primarily responsible for the spectroscopic properties of the solute. It is convenient to separate $X$ into a constant part and a fluctuation,

$$
X=\langle X\rangle+\Delta X
$$

where the average is taken over a thermal equilibrium distribution in the ground electronic state of the solute,

$$
\langle\cdots\rangle=\operatorname{Tr}\left[\ldots \rho_{0}\right], \quad \rho_{0}=e^{-\beta H_{g}} \operatorname{Tr} e^{-\beta H_{g}}, \quad \beta=1 / k_{B} T .
$$

For the model in Eqs. (1) and (2) the average value of $X$ is equal to

$$
\langle X\rangle=\Delta U+\lambda .
$$

\section{DYNAMIC STOKES SHIFT CALCULATION}

\section{A. Instantaneous pump pulse}

As noted earlier it is usually assumed that the solvent state does not change during the photoinduced electronic excitation of the solute molecule. A sudden change of the electronic state of the molecule can then be viewed as switching on the potential $X$ at $t=0$. The constant energy change, $\langle X\rangle$, which does not influence the solvent dynamics, can be omitted. The average value [averaged as in Eq. (22) given below] of a dynamical variable will be denoted by the bar over that variable. The average value of the solvation coordinate variation $\Delta X$ can then be obtained as a linear response to the "'applied external force," which is a unit step function $-\theta(t)(\theta(t)=0$ if $t<0$, and 1 for $t>0)$,

$$
\overline{\Delta X}(t)=-\int_{-\infty}^{t} \alpha\left(t-t^{\prime}\right) \theta\left(t^{\prime}\right) d t^{\prime}=-\int_{0}^{t} \alpha(\tau) d \tau .
$$

The generalized susceptibility $\alpha(t)$ is given in linear response theory in terms of a correlation function of the solvation coordinate, ${ }^{66}$

$$
\alpha(t)=-\frac{1}{i \hbar}\left\langle\left[\Delta X_{g}(t), \Delta X_{g}(0)\right]\right\rangle,
$$

where the square brackets denote the commutator, the subscript $g$ in $\Delta X_{g}$ denotes a dynamical evolution of $\Delta X$ that proceeds with the Hamiltonian $H_{g}$, i.e., $\Delta X_{g}(t)$ $=\exp \left(i H_{g} t / \hbar\right) \Delta X \exp \left(-i H_{g} t / \hbar\right)$, and the thermal averaging is 
performed using the ground electronic state of the solute, as in Eq. (6). Introducing a correlation function of the solvation coordinate,

$$
C(t)=\left\langle\Delta X_{g}(t) \Delta X_{g}(0)\right\rangle,
$$

Eq. (9) can be rewritten as ${ }^{67}$

$$
\alpha(t)=-\frac{2}{\hbar} \operatorname{Im}[C(t)] .
$$

Equations (8)-(11) are valid for arbitrary molecular Hamiltonians $H_{g}$ and $H_{e}$ but assuming a linear response approximation. It is difficult to formulate a rigorous, quantitative criterion of applicability of linear response theory, and do not presuppose any molecular harmonic oscillator model. However, numerical simulations ${ }^{1,4,37,68-73}$ have shown that in most cases for all but very small solutes the linear response theory is applicable even for values of $\Delta X$ which are much larger than its thermal fluctuation. Equation (9) is also applicable in classical mechanics, in the same approximation, when the commutator $[\ldots, \ldots] / i \hbar$ is interpreted as the Poisson bracket. ${ }^{66}$

To characterize the time-evolution of the fluorescence spectrum the mean optical frequency $\nu(t)$ at time $t$ could be used, ${ }^{74}$

$$
h \nu(t)=\bar{X}(t),
$$

where $h=2 \pi \hbar$ is Planck's constant. The frequency $\nu$ depends on the properties of the solute molecule as well as on those of the solvent. Commonly, instead, a dimensionless solvent response function $S(t)$ is used to characterize the solvent-related aspect of the Stokes shift evolution, ${ }^{48}$

$$
S(t)=\frac{\nu(t)-\nu(\infty)}{\nu(0)-\nu(\infty)} .
$$

In classical mechanics Eq. (9) is substituted by ${ }^{66}$

$$
\alpha(t)=-\beta \frac{d}{d t} C_{\mathrm{cl}}(t),
$$

where $C_{\mathrm{cl}}(t)$ is the classical correlation function of the solvation coordinate

$$
C_{\mathrm{cl}}(t)=\left\langle\Delta X_{g}(t) \Delta X_{g}(0)\right\rangle_{\mathrm{cl}} .
$$

In the latter the averaging is over the equilibrium classical statistical ensemble appropriate to the ground electronic state of the solute. Substituting Eq. (14) into Eq. (8) and then into Eq. (13) one obtains

$$
S(t)=C_{\mathrm{cl}}(t) / C_{\mathrm{cl}}(0) .
$$

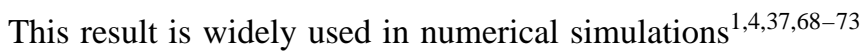
to describe the time-evolution of the Stokes shift in terms of the classical correlation function of the solvation coordinate [cf. Eqs. (8) and (19)]. It does not assume that the motion is harmonic.

For the harmonic oscillator model in Eqs. (1) and (2) the correlation function of the solvation coordinate, Eq. (10), is given by ${ }^{75}$

$$
C(t)=\hbar \sum_{j} \frac{c_{j}^{2}}{2 \omega_{j}}\left[\operatorname{coth}\left(\beta \hbar \omega_{j} / 2\right) \cos \omega_{j} t-i \sin \omega_{j} t\right] .
$$

In the classical limit, $\hbar \rightarrow 0$, Eq. (17) is reduced to the following expression:

$$
C_{\mathrm{cl}}(t)=\beta^{-1} \sum_{j} \frac{c_{j}^{2}}{\omega_{j}^{2}} \cos \omega_{j} t .
$$

From Eqs. (11) and (17) one obtains $\alpha(t)$ for the harmonic oscillator model:

$$
\alpha(t)=\sum_{j} \frac{c_{j}^{2}}{\omega_{j}} \sin \omega_{j} t .
$$

Substituting Eq. (19) into Eq. (8) and then into Eq. (13) one obtains

$$
S(t)=\Delta(t),
$$

where

$$
\Delta(t)=\frac{1}{2 \lambda} \sum_{j} \frac{c_{j}^{2}}{\omega_{j}^{2}} \cos \omega_{j} t .
$$

The function $\Delta(t)$, which coincides with the normalized classical correlation function $C_{\mathrm{cl}}(t)$ [cf. Eq. (16)], vanishes when $t \rightarrow \infty$, and, as one can see from its definition and Eq. (4), equals unity at $t=0$.

Equations (16) and (20) allow one to relate the solvent response function, Eq. (13), to the correlation function, Eq. (15), of the solvation coordinate for an instantaneous pump pulse. Equation (16), which is valid for a generic nonlinear system, looks more general than Eq. (20). It is worth noting, however, that if one defines coefficients $c_{j}$ of the effective harmonic oscillator model in Eq. (2) in such a way that the correlation function $C_{\mathrm{cl}}(t)$, Eq. (15), of the nonlinear system is fitted with suitable choice of $c_{j}$ 's to Eq. (18), then Eqs. (16) and (20) will be identical (see the discussion below).

We are not aware of any success in generalizing the above procedure to the case where the electronic transition of the molecule cannot be viewed as instantaneous. Accordingly, we describe next a different approach, a density matrix method which can be used to treat the solvent dynamics for the case of an arbitrary duration of the pump pulse. Conceptually, it is close to the method used by Mukamel and coworkers, ${ }^{51-53}$ but the execution is different. When the pulse is instantaneous the results will be shown to reduce to those given by the previous method, Eqs. (8) and (19).

\section{B. Pump pulse of finite duration}

We introduce the density matrix of the solvent $\rho(t)$, which is evolving on a potential energy surface involving the excited electronic state of the solute. The average value of the solvation coordinate $\bar{X}(t)$ at the time $t$ after the excitation can then be written as

$$
\bar{X}(t)=\operatorname{Tr}[X \rho(t)],
$$

where $\rho(t)=\exp \left(-i H_{e} t / \hbar\right) \rho(0) \exp \left(i H_{e} t / \hbar\right)$. 
This expression for $\bar{X}(t)$ can be rewritten in an equivalent form:

$$
\bar{X}(t)=\operatorname{Tr}\left[X_{e}(t) \rho(0)\right],
$$

where the subscript $e$ means that the evolution of $X$ proceeds with the Hamiltonian $H_{e}, X_{e}(t)=\exp \left(i H_{e} t / \hbar\right) X$ $\times \exp \left(-i H_{e} t / \hbar\right)$. It is shown in Appendix A that for the model in Eqs. (1) and (2) the following relation is satisfied:

$$
X_{e}(t)=X_{g}(t)+2 \lambda[\Delta(t)-1],
$$

where $\Delta(t)$ is given by Eq. (21).

For comparison with our later results we first consider the case that the density matrix $\rho(t)$ of the solvent does not change during the electronic transition ("instantaneous pump pulse'"). In this case $\rho(0) \simeq \rho_{0}$, the equilibrium solvent distribution corresponding to the ground electronic state of the solute [cf. Eq. (6)]. Using Eqs. (7), (23) and (24) one immediately obtains

$$
\bar{X}(t)=\Delta U+\lambda[2 \Delta(t)-1],
$$

and, as a consequence, obtains Eq. (20) upon using Eqs. (12) and (13).

For a pump pulse of finite duration the actual density matrix of the solvent $\rho$ immediately after electronic excitation of the solute will differ from the $\rho_{0}$ defined in Eq. (6). To calculate the density matrix $\rho(t)$ the process of the excitation now needs to be considered explicitly. To this end we introduce the common assumptions that the pump pulse radiation field $E(t)$ can be described classically and that the dipole approximation can be used for its interaction with the solute, ${ }^{51}$

$$
H_{\mathrm{int}}(t)=\frac{E(t)+E^{*}(t)}{2} \hat{\mu}, \quad \hat{\mu}=\mu(|e\rangle\langle g|+| g\rangle\langle e|),
$$

where the electric field is treated as linearly polarized along the $x$-axis $E(t)=E_{x}(t)$. It is also assumed that $E(t)$ has a relatively narrow spectrum, so one can write it in a quasiharmonic form,

$$
E(t)=E_{0}(t) \exp \left(-i 2 \pi \nu_{0} t\right),
$$

where $E_{0}(t)$ is a function changing slowly with time. The asterisk in Eq. (26) denotes the complex-conjugate.

The transition dipole moment of the solute along the $x$ axis is $\mu=\mu_{0} n_{x}$, where $n_{x}$ is the directional cosine of the transition dipole moment along the $x$ axis, and is treated as a constant. It may be noted that the last assumption is usually referred to a spatially fixed solute (Condon approximation) and, strictly speaking, is not applicable to a moving solute. However, one dynamical effect, that of the solute reorientation, which is frequently modeled as rotational diffusion, ${ }^{48}$ is rather small on a time scale of the processes considered in the present article, especially for large dye solute molecules which are commonly used in these experiments and whose orientational diffusion is relatively slow: A relative change of the transition dipole moment due to the solute diffusional reorientation can be estimated as $\delta \mu / \mu_{0} \sim \sqrt{D t}$, where $D$ is the rotational diffusion coefficient and $t$ is the relaxation time. Substituting an estimate from Ref. 48, $D=5$ $\times 10^{8} \mathrm{~s}^{-1}$ and taking $t=1 \mathrm{ps}, \delta \mu / \mu_{0}$ is estimated to be of the order of $2 \%$ which is probably at least as good as the other approximations. Thereby, the effect of the solute reorientation can be treated statically, averaging the final result over all possible solute orientations at the end of the calculation.

The orientation of the solute influences only the amplitude of the corresponding perturbation Hamiltonian, both for the excitation pulse [cf. Eq. (26)] and for the resulting fluorescence spectrum. ${ }^{52}$ As a result, the solute orientation does not influence the shape of the transient fluorescence spectrum but only its directional properties. The dependence of the fluorescence intensity on the direction of observation and on the fluorescence polarization direction is considered in Appendix B for completeness.

To find the density matrix of the solvent $\rho(t)$ with the solute in the excited electronic state, second-order timedependent perturbation theory with $H_{\text {int }}$ as a perturbation must be used. Under the rotating wave approximation, the expression for $\rho(t)$ is given by ${ }^{12}$

$$
\begin{aligned}
\rho(t)= & \frac{\mu^{2}}{4 \hbar^{2}} \int_{-\infty}^{+\infty} \int_{-\infty}^{+\infty} d t^{\prime} d t^{\prime \prime} E^{*}\left(t^{\prime}\right) E\left(t^{\prime \prime}\right) \\
& \times e^{-i\left(t-t^{\prime \prime}\right) H_{e} / \hbar} e^{-i t^{\prime \prime} H_{g} / \hbar} \rho_{0} e^{i t^{\prime} H_{g} / \hbar} e^{i\left(t-t^{\prime}\right) H_{e} / \hbar} .
\end{aligned}
$$

Here and below we assume that the fluorescence signal is observed when the pump pulse is already over. If, instead, the pump and upconverting pulses overlap, the observed signal cannot be interpreted as a pure fluorescence, but contains also a Raman scattering component. ${ }^{12}$ The $\rho(t)$ in Eq. (28) is a part of the "solvent + solute" system total density matrix, which is diagonal over the excited electronic state of the solute. Since we neglect nonradiative electronic transitions of the solute, the time-evolution of $\rho(t)$ can be considered separately. We will normalize $\rho(t)$ for convenience. The normalized $\rho(t)$, i.e., such that $\operatorname{Tr}[\rho(t)]=1$, is given by

$$
\begin{aligned}
\rho(t)= & \frac{1}{v_{0}} \int_{-\infty}^{+\infty} \int_{-\infty}^{+\infty} d t^{\prime} d t^{\prime \prime} E^{*}\left(t^{\prime}\right) E\left(t^{\prime \prime}\right) \\
& \times e^{-i\left(t-t^{\prime \prime}\right) H_{e} / \hbar} e^{-i t^{\prime \prime} H_{g} / \hbar} \rho_{0} e^{i t^{\prime} H_{g} / \hbar} e^{i\left(t-t^{\prime}\right) H_{e} / \hbar},
\end{aligned}
$$

where

$$
\begin{aligned}
& v_{0}=\int_{-\infty}^{+\infty} \int_{-\infty}^{+\infty} d t^{\prime} d t^{\prime \prime} E^{*}\left(t^{\prime}\right) E\left(t^{\prime \prime}\right) R\left(t^{\prime}-t^{\prime \prime}\right), \\
& R(\tau)=\left\langle e^{-i \tau H_{e} / \hbar} e^{i \tau H_{g} / \hbar}\right\rangle,
\end{aligned}
$$

where $\langle\cdots\rangle$ denotes thermal average, Eq. (6). The function $R(\tau)$ coincides with the normalized correlation function of the operator for the transition dipole moment $\hat{\mu}$, and its Fourier transform gives the absorption lineshape ${ }^{76}$ The correlation function $R(\tau)$ is expressed in terms of the (quantum) correlation function of the solvation coordinate, Eq. (10), as $^{76}$ 


$$
R(\tau)=\exp \left[-\frac{i}{\hbar} \tau\langle X\rangle-\frac{1}{\hbar^{2}} \int_{0}^{\tau} d \tau^{\prime} \int_{0}^{\tau^{\prime}} C\left(\tau^{\prime}-\tau^{\prime \prime}\right) d \tau^{\prime \prime}\right]
$$

If the pulse is infinitely short, which formally corresponds to using $E(t) \propto \delta(t)$, then $\rho(t) \rightarrow \rho(0)=\rho_{0}$, at $t \rightarrow+0$, a situation discussed above.

Equations (22), (12), (13), and (29)-(32) provide a basis for calculating the time-evolution of the Stokes shift for an arbitrary pump pulse. Using Eq. (29) the average value of the solvent coordinate $X$ at time $t$ can be represented in the form:

$$
\bar{X}(t)=\frac{1}{v_{0}} \int_{-\infty}^{+\infty} \int_{-\infty}^{+\infty} d t^{\prime} d t^{\prime \prime} f\left(t \mid t^{\prime}, t^{\prime \prime}\right) E^{*}\left(t^{\prime}\right) E\left(t^{\prime \prime}\right),
$$

where the integral kernel $f\left(t \mid t^{\prime}, t^{\prime \prime}\right)$ is given by

$$
f\left(t \mid t^{\prime}, t^{\prime \prime}\right)=\operatorname{Tr}\left[X_{e}\left(t-t^{\prime}\right) e^{-i\left(t^{\prime}-t^{\prime \prime}\right) H_{e} / \hbar} e^{i\left(t^{\prime}-t^{\prime \prime}\right) H_{g} / \hbar} \rho_{0}\right] .
$$

A straightforward but somewhat cumbersome calculation given in Appendix $\mathrm{C}$ yields the following expression for this kernel:

$$
\begin{aligned}
f\left(t \mid t^{\prime}, t^{\prime \prime}\right)= & R\left(t^{\prime}-t^{\prime \prime}\right)\left\{\lambda\left[\Delta\left(t-t^{\prime}\right)+\Delta\left(t-t^{\prime \prime}\right)\right]-\lambda+\Delta U\right. \\
& +i \sum_{j} \frac{c_{j}^{2}}{\omega_{j}^{2}} \operatorname{coth}\left(\beta \hbar \omega_{j} / 2\right) \sin \left[\omega_{j}\left(t^{\prime \prime}-t^{\prime}\right) / 2\right] \\
& \left.\times \cos \left[\omega_{j}\left(t-t^{\prime \prime} / 2-t^{\prime} / 2\right)\right]\right\} .
\end{aligned}
$$

It is easily seen that in the short pump pulse limit Eq. (35) reduces to a previous result, Eq. (25). Really, in this limit the integration times $t^{\prime}$ and $t^{\prime \prime}$ can be set to zero in all terms in braces. As a result, the expression for $f$ takes a simple form: $f\left(t \mid t^{\prime}, t^{\prime \prime}\right)=R\left(t^{\prime}-t^{\prime \prime}\right)[2 \lambda \Delta(t)-\lambda+\Delta U]$. Upon substituting this expression into Eq. (33), Eq. (25) immediately follows.

In the harmonic oscillator model the solute-solvent interaction is characterized by the "normal mode shifts" $c_{j}$ in Eq. (2). Physically important, however, are not so much the $c_{j}$ 's themselves but their combination in the well known form, the spectral density function $J(\omega)$ of the solvent modes, ${ }^{54}$

$$
J(\omega)=\frac{\pi}{2} \sum_{j}\left(c_{j}^{2} / \omega_{j}\right) \delta\left(\omega-\omega_{j}\right),
$$

where $\delta(\omega)$ denotes the Dirac delta function. Using the spectral density function allows one most naturally go to the limit $N=\infty$. If the number of harmonic modes is finite then $J(\omega)$ is the sum of finite number of delta functions. In the limit $N \rightarrow \infty J(\omega)$ is transformed to a regular continuous function. Using the definition of the spectral density function, the expressions for the reorganization energy $\lambda$, for $\Delta(t)$, and for the correlation function $C(t)$, Eqs. (4), (21), and (17), can be written as

$$
\lambda=\frac{1}{\pi} \int_{0}^{\infty} d \omega \frac{J(\omega)}{\omega}, \Delta(t)=\frac{1}{\pi \lambda} \int_{0}^{\infty} d \omega \frac{J(\omega)}{\omega} \cos \omega t,
$$

and

$$
C(t)=\frac{\hbar}{\pi} \int_{0}^{\infty} d \omega J(\omega)[\operatorname{coth}(\beta \hbar \omega / 2) \cos \omega t-i \sin \omega t] .
$$

Equation (35) can be rewritten using the definition of $J(\omega)$ as

$$
\begin{aligned}
f\left(t \mid t^{\prime}, t^{\prime \prime}\right)= & R(\tau)\{\lambda[\Delta(t-T-\tau / 2)+\Delta(t-T+\tau / 2)] \\
& -\lambda+\Delta U+-i \frac{2}{\pi} \int_{0}^{\infty} d \omega \frac{J(\omega)}{\omega} \operatorname{coth}(\beta \hbar \omega / 2) \\
& \times \sin (\omega \tau / 2) \cos \omega(t-T)\}
\end{aligned}
$$

where we have also changed the integration variables:

$$
\tau=t^{\prime}-t^{\prime \prime}, \quad T=\left(t^{\prime \prime}+t^{\prime}\right) / 2 \text {. }
$$

Equation (39) has been derived using the harmonic oscillator model, Eqs. (1) and (2). However, as was noted in the introduction in Sec. II, this equation and other equations which follow from it have a broader validity and can be applied to a nonlinear system too. To this end, one has to redefine the spectral density function $J(\omega)$, which occurs in Eq. (39), because Eq. (36), which was used as a definition of $J(\omega)$, is no longer valid for the nonlinear system. The easiest way of doing this, leading to Eq. (41) below, is to use the harmonic oscillator model to relate the spectral density function to the imaginary part of the quantum correlation function of the solvation coordinate, Eq. (38). (It is important to use a quantum correlation function because for a generic nonlinear system, in contrast to a harmonic one, there is no simple relation between the classical and quantum correlation functions.) Applying the inverse Fourier transform to the imaginary part of Eq. (38) one obtains:

$$
J(\omega)=\frac{2}{\hbar} \int_{0}^{\infty} \operatorname{Im}[C(t)] \sin \omega t d t,
$$

where $C(t)$ is given by Eq. (10). Equation (39), with $J(\omega)$ given by Eq. (41), can be derived more generally following Mukamel's type of argument, ${ }^{51}$ without introducing any molecular harmonic oscillator model.

To proceed further analytically with Eqs. (33) and (39) we assume for a moment that the correlation function $C(t)$ which enters into $R(t)$, Eq. (32), can be approximated by its value at zero time,

$$
C(t) \simeq C(0) \text {. }
$$

While this approximation is always qualitatively correct, it neglects the important contribution to the absorption spectrum which arises from the solute's high-frequency vibrational modes. These effects will be taken into account later in Eqs. (56) and (58).

Substituting Eq. (42) into Eq. (32) one obtains: 


$$
R(\tau)=\exp \left[-C(0) \tau^{2} / 2 \hbar^{2}-i\langle X\rangle \tau / \hbar\right],
$$

where $\langle X\rangle$ is given by Eq. (7). Assuming that the main contribution to the correlation function $C(t)$, Eq. (38), arises from low frequency modes (classical modes) one can use an estimate for $C(0)$ [cf. Eqs. (37) (38)],

$$
C(0) \sim 2 \lambda / \beta \text {. }
$$

Equations (43) and (44) define the important time-scale $\tau_{c}$ over which the correlation function $R(\tau)$, Eq. (31), is essentially different from zero:

$$
\tau_{c}=\hbar \sqrt{\beta / \lambda} .
$$

The correlation function $R(\tau)$ limits the important time difference $\tau$ in Eq. (39) to being less than $\tau_{c}$. Within such times the sine under the integral in Eq. (39) can be replaced by its argument $\omega \tau / 2$ and $\tau$ can also be neglected in the arguments of the functions $\Delta(t-T \pm \tau / 2)$ giving as a result:

$$
\begin{aligned}
f\left(t \mid t^{\prime}, t^{\prime \prime}\right)= & R(\tau)\{2 \lambda \Delta(t-T)-\lambda \\
& \left.+\Delta U-i \hbar^{-1} \tau \operatorname{Re}[C(t-T)]\right\},
\end{aligned}
$$

where we have used the expression for $C(t)$, Eq. (38). Substituting Eq. (46) and a quasi-harmonic representation for the pump pulse, Eq. (27), into Eqs. (30) and (33) one arrives at the following expression for $\bar{X}(t)$ :

$$
\bar{X}(t)=\frac{\int_{-\infty}^{\infty} d T\left|E_{0}(T)\right|^{2} K(t-T)}{\int_{-\infty}^{\infty} d T\left|E_{0}(T)\right|^{2}},
$$

where

$$
K(t)=\frac{\int_{-\infty}^{\infty} d \tau \exp \left(i 2 \pi \nu_{0} \tau\right) R(\tau)\left\{2 \lambda \Delta(t)-\lambda+\Delta U-i \hbar^{-1} \tau \operatorname{Re}[C(t)]\right\}}{\int_{-\infty}^{\infty} d \tau \exp \left(i 2 \pi \nu_{0} \tau\right) R(\tau)} .
$$

In derivation of Eqs. (47) and (48) we again neglected the small time difference $\tau$ in $E_{0}(T \pm \tau / 2)$, which changes slowly with time. The last assumption implies that the pulse duration $\tau_{p}$ is much longer than the correlation time $\tau_{c}$, Eq. (45),

$$
\tau_{p} \gg \tau_{c} .
$$

Equation (49) is typically satisfied for a system at a room temperature with strong solute-solvent interaction and for a pump pulse with $\tau_{p} \geqslant 50 \mathrm{fs}$.

From Eq. (47) one can see that the DSS resulting from a long pump pulse is given by convolution of the pulse shape and the function $K(t)$ given by Eq. (48). This function describes a DSS which corresponds to a pulse which is much longer than the correlation time $\tau_{c}$, Eq. (45), but still shorter than any time scale, relevant to the solvent dynamics. To calculate $K(t)$ we first use the Gaussian approximation for $R(\tau)$, Eq. (43). Substituting Eq. (43) into Eq. (48) and integrating over $\tau$ one obtains

$$
K(t)=\Delta U-\lambda+2 \lambda \Delta(t)+h \Delta \nu_{0} \Delta_{1}(t),
$$

where $\Delta \nu_{0}$ is the central frequency shift of the pump pulse $\nu_{0}$ relative to the maximum of the absorption spectrum,

$$
\Delta \nu_{0}=\nu_{0}-(\Delta U+\lambda) / h \text {. }
$$

The function $\Delta_{1}(t)$ is the quantum analog of the normalized classical correlation function $\Delta(t)$ [Eq. (37)],

$$
\Delta_{1}(t)=\frac{\operatorname{Re}[C(t)]}{C(0)},
$$

$\Delta_{1}(0)=1$, and $C(t)$ is given by Eq. (38). It is convenient for comparison later with Eq. (73) to rewrite the DSS for a short pulse, Eq. (50), in a different form:

$$
\bar{X}(t)-\bar{X}(\infty)=2 \lambda \Delta(t)+h \Delta \nu_{0} \Delta_{1}(t) .
$$

It can be seen from this equation that the variation of the transition frequency $\Delta \nu=\nu(0)-\nu(\infty)=[\bar{X}(0)-\bar{X}(\infty) / h$ is:

$$
\Delta \nu=\Delta \nu_{0}+2 \lambda / h \text {. }
$$

The solvent response function $S(t)$ is obtained by substituting Eq. (50) into Eq. (13):

$$
S(t)=\frac{1}{1+\kappa}\left[\Delta(t)+\kappa \Delta_{1}(t)\right], \quad \kappa=\frac{h \Delta \nu_{0}}{2 \lambda} .
$$

Equation (55) gives a simple expression for the solvent response function which generalizes Eq. (20) and reduces to it when $\hbar \rightarrow 0$ or when pump pulse is not off-resonance $\left(\Delta \nu_{0}=0\right)$. The expression in Eq. (55) with $\kappa=0$ corresponds to the purely classical response of the solvent [cf. Eq. (20)]. The quantum correlation function $\Delta_{1}(t)$ and $\kappa$ are responsible for the quantum effects entering into the solvent response. For a choice of $h \Delta \nu_{0} \sim 2 \sqrt{\lambda / \beta}$, which is the absorption linewidth, one can estimate the contribution of $\kappa$ to the total solvent response as $\kappa \sim 1 / \sqrt{\lambda \beta}$, which is typically small. However, particularly in the far wing on the red side a larger $\Delta \nu_{0}$ can be used. We give an interpretation of Eq. (55) later. We note that Eqs. (53) and (55) contain no properties specific to a molecular harmonic oscillator model.

A Gaussian approximation for the correlation function $R(t)$ [use of Eq. (43) to represent Eq. (31)] may be too restrictive for a solute with a complex spectrum. Using Eq. (48) one readily obtains the following expression for the DSS:

$$
\begin{aligned}
K(t)= & \Delta U-\lambda+2 \lambda \Delta(t) \\
& -\left.\hbar^{-1} C(0) \frac{d \ln [\widetilde{R}(\omega)]}{d \omega}\right|_{\omega=2 \pi \nu_{0}} \Delta_{1}(t),
\end{aligned}
$$

where $\widetilde{R}(\omega)$ is the Fourier transform of $R(t)$, 


$$
\widetilde{R}(\omega)=\int_{-\infty}^{\infty} e^{i \omega t} R(t) d t
$$

The absorption coefficient is proportional to $\omega \widetilde{R}(\omega){ }^{76}$ It follows from Eq. (56) that for a solute with a non-Gaussian absorption spectrum, even if the solvent dynamics can be described classically, i.e., if $\Delta_{1}(t) \simeq \Delta(t)$, then the solvent response function $S(t)$ is given by Eq. (20), but the variation of the transition frequency $\nu(0)-\nu(\infty)$ will differ from that predicted from the classical theory, $\Delta \nu_{0}+2 \lambda / h$ [cf. Eq. (54)]. A generalization of the expression for $\kappa$ in Eq. (55) for a solute with an arbitrary absorption spectrum is:

$$
\kappa=-\left.\frac{C(0)}{2 \hbar \lambda} \frac{d \ln [\widetilde{R}(\omega)]}{d \omega}\right|_{\omega=2 \pi \nu_{0}} .
$$

One aim in the present paper is to estimate for a realistic experimental situation the change in the Stokes shift timeevolution due to the finite pump pulse duration. To estimate the Stokes shift dynamics a realistic spectral density function $J(\omega)$ in Eq. (41) is needed for the solute-solvent interaction. The main contribution to the interaction of polar solutes with small-molecule polar solvents is due to long range dipoledipole and charge-dipole interactions, together with hydrogen bonding in the case of protic solvents. ${ }^{77-79}$ Frequently, the solute-solvent interaction in polar solvents has been described in terms of continuum models using an exponential or multiexponential dielectric response. ${ }^{5,48,80}$ It has been argued by some researchers that due to inherent molecular nature of the solvation process, the continuum models ultimately fail to explain some important features of solvation, in particular its initial, ultrafast stage. In more recent investigations, however, it was found that once one includes not only the low-frequency, diffusional part of the solvent's dielectric response but also the high-frequency, inertial part, a prominent role of the inertial motion in solvation is recovered. ${ }^{36,81,82}$ In their work on the dynamic Stokes shift of coumarin 343 anion (C343) in water, Hsu et al. ${ }^{82}$ obtained encouraging agreement with the experiment ${ }^{2}$ upon using the experimental dielectric response function $\epsilon(\omega)$ for water and a continuum-based approach.

In the present paper we again use for simplicity the Onsager model for the solute, which treats the solute as a dipole in the center of a spherical cavity and the solvent as a dielectric continuum with uniform properties, surrounding the solute. The dielectric response of the solvent is assumed to be local and to be characterized by the experimental bulk dielectric response function $\epsilon(\omega)$. The spectral density $J(\omega)$ of the solvent's normal modes can be related to the dielectric function using the expression for the DSS caused by an instantaneous pump pulse. Such a DSS can readily be expressed in terms of the spectral density function using Eqs. (25) and (37),

$$
\bar{X}(t)=\Delta U-\lambda+\frac{2}{\pi} \int_{0}^{\infty} d \omega \frac{J(\omega)}{\omega} \cos \omega t .
$$

On the other hand, within the framework of the reaction field approach the DSS associated to an instantaneous pump pulse is given by, ${ }^{82}$

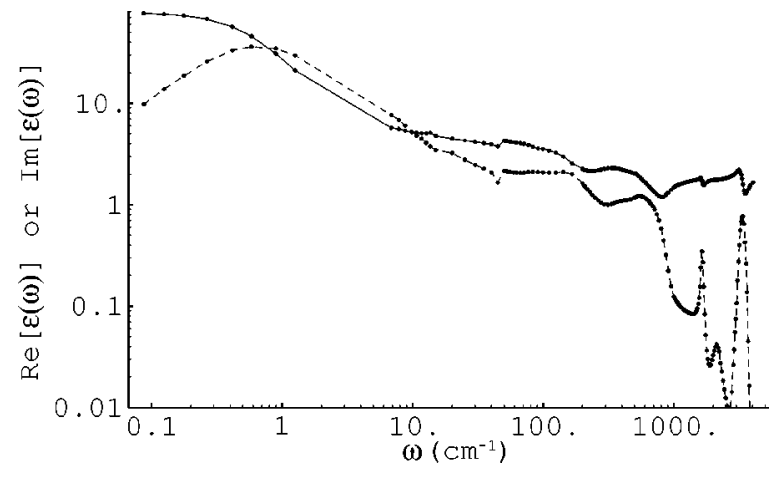

FIG. 1. The real and imaginary parts of the dielectric susceptibility of water as functions of frequency (Refs. 83-86).

$$
\bar{X}(t)=\Delta U-\lambda-\frac{4 \Delta \mu^{2}}{a^{3} \pi} \int_{0}^{\infty} d \omega \frac{\cos \omega t}{\omega} \operatorname{Im}\left[\frac{\epsilon(\omega)-1}{2 \epsilon(\omega)+1}\right],
$$

where $\Delta \mu=\mu_{e}-\mu_{g}$ is the (vector) difference of the dipole moments in the excited and ground electronic states, $a$ is the solute cavity radius, and the minus sign in Eq. (60) appears when the convention is used that the imaginary part of the dielectric function $\epsilon(\omega)$ is taken to be negative. Comparing Eqs. (59) and (60) one obtains the desired expression:

$$
J(\omega)=-\frac{2 \Delta \mu^{2}}{a^{3}} \operatorname{Im}\left[\frac{\epsilon(\omega)-1}{2 \epsilon(\omega)+1}\right] .
$$

It can be shown by other methods ${ }^{49,52}$ using linear response theory that Eq. (61) holds in most general conditions for the Onsager model if one assumes that the dielectric response of the solvent is local on atomic length-scale.

Using Eq. (61) the solvation dynamics of the system can be estimated once the dielectric function of the solvent $\epsilon(\omega)$ is known. As an example the dielectric dispersion data for water at $T=298 \mathrm{~K}$ (Fig. 1) were used to calculate the spectral density of the solvent modes. Water has been used in DSS measurements ${ }^{2,42}$ and its dielectric response function is available at a high level of accuracy over a wide range of frequencies. ${ }^{83-86}$ Equation (55) shows that the effect of the finite pump pulse duration on the DSS depends on the difference between $\Delta(t)$ and $\Delta_{1}(t)$, Eqs. (37) and (52). These functions are shown in Fig. 2. A relative contribution of the ultrafast component to the correlation function $\Delta_{1}(t)$ is larger due to the $0 \mathrm{~K}$ fluctuations of the quantum modes, and the oscillations with the period of $10-15$ fs are much stronger. The exponential relaxation time (appropriate in the low frequency regime) is the same for both $\Delta(t)$ and $\Delta_{1}(t)$. This result is expected since the long time scale orientational relaxation is associated with the slow classical solvent modes.

The instrument response time (FWHM of a crosscorrelation of the pump and gate pulses ${ }^{87}$ ), which characterizes the time-resolution in a measurement of the transient fluorescence also must be taken into account. It is not better than about $100 \mathrm{fs}^{36}$ The correlation functions $\Delta(t)$ and $\Delta_{1}(t)$ were next convoluted with appropriate Gaussian shapes of both the pump pulse [cf. Eq. (47)] and the upconverting pulse, ${ }^{88}$ with the results given in Fig. 3. These con- 


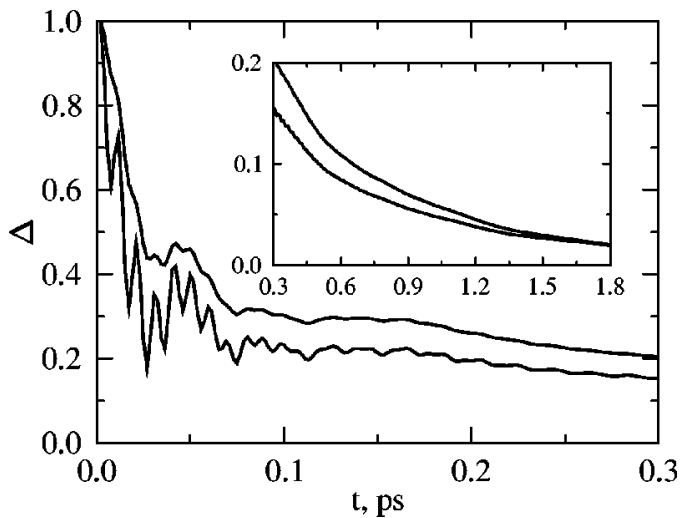

FIG. 2. The normalized correlation functions $\Delta(t)$ [Eq. ( 37)] and $\Delta_{1}(t)$ [Eq. (52)] are given by the upper and lower curves, respectively. The inset gives the results over a longer picosecond time interval. The spectral density of the solvent was calculated using the dielectric data of water and Eq. (61).

volutions make the difference between the classical and quantum correlation functions even less pronounced. Taking into account the fact that $\Delta_{1}(t)$ enters into the total solvent response $R(t)$ with the weight $\kappa$ [Eq. (55)], which is generally much less than unity, one can conclude that the deviation from the standard formula [Eq. (20)] due to the finite pump pulse duration is small and can be neglected in most DSS experiments. Some deviation can be expected when the central frequency of the pump pulse lies in the far wing of the absorption band of the chromophore and, then the additional contribution in the correlation function $\Delta_{1}(t)$ to the total solvent response can be comparable with the standard term, $\Delta(t)$. For example, a red shift of the pump pulse from the fluorescence maximum, $\Delta \nu_{0} \simeq \lambda / h$, which would give the excitation probability of the order of $10 \%$ of the maximum for Coumarin 153 in ethanol, leads to $\kappa \sim 0.5$.

\section{PHYSICAL INTERPRETATION OF EQS. (53) AND} (55)

To interpret Eq. (53) we first obtain, in Eqs. (62)-(65) below, the distribution of the displacements $Q_{j}$ of the harmonic oscillators before and immediately after the electronic

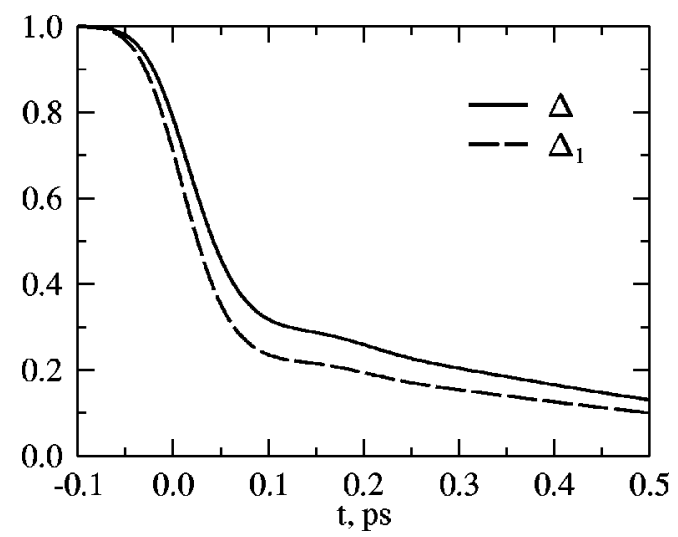

FIG. 3. The correlation functions $\Delta(t)$ and $\Delta_{1}(t)$ with finite time resolution. Convolution was performed with the Gaussian $\exp \left(-t^{2} / \tau_{p}^{2}\right), \tau_{p}=50 \mathrm{fs}$, which corresponds to an instrument response function of $\mathrm{FWHM}=$ $2 \sqrt{\ln 2} \tau_{p}=83$ fs (Ref. 88). transition. The probability distribution $W_{j}$ of the coordinate $Q_{j}$ for a single oscillator in equilibrium with the ground electronic state of the solute (the statistical state of the system before the excitation) is given by ${ }^{89}$

$$
\begin{aligned}
W_{j}\left(Q_{j}\right)= & \sqrt{\frac{\omega_{j} \tanh \left(\hbar \omega_{j} \beta / 2\right)}{\pi \hbar}} \\
& \times \exp \left[-\tanh \left(\hbar \omega_{j} \beta / 2\right) \omega_{j} Q_{j}^{2} / \hbar\right] .
\end{aligned}
$$

In the high temperature (low frequency) limit this distribution reduces to the classical one:

$$
W_{j}\left(Q_{j}\right) \simeq \sqrt{\frac{\omega_{j}^{2} \beta}{2 \pi}} \exp \left(-\beta \omega_{j}^{2} Q_{j}^{2} / 2\right), \quad \hbar \omega_{j} \beta / 2 \ll 1 .
$$

In the low temperature (high frequency) limit it reduces to the probability distribution corresponding to the ground state of the oscillator:

$$
W_{j}\left(Q_{j}\right) \simeq \sqrt{\frac{\omega_{j}}{\pi \hbar}} \exp \left(-\omega_{j} Q_{j}^{2} / \hbar\right), \hbar \omega_{j} \beta / 2 \gg 1,
$$

which is much broader than the classical distribution when $\hbar \omega_{j} \beta / 2 \gg 1$. The last property can be interpreted as the result of nuclear tunneling of the oscillator to nonclassical regions. The total distribution of all oscillators representing the solvent is given by the product of the distributions in Eq. (62),

$$
W(\mathbf{Q})=\prod_{j} W_{j}\left(Q_{j}\right)
$$

The maximum of the absorption spectrum corresponds to $Q_{j}=0$. If the pump pulse frequency is tuned away from the maximum of the absorption spectrum the mean displacements $\overline{Q_{j}^{0}}$ of the $j$ th harmonic oscillator excited by this offresonance optical excitation immediately after the excitation deviate from their initial zero values. To find these displacements one must maximize the probability $W(\mathbf{Q})$ in Eq. (65) subject to the constraint that the frequency shift $\Delta \nu_{0}$ is kept fixed. Equations (3), (12), and (51) yield for this constrain:

$$
h \Delta \nu_{0}=\sum_{j} c_{j} Q_{j}
$$

Using the $\log W(\mathbf{Q})$ as a function to be maximized and applying to it the method of Lagrange multipliers we have,

$$
\frac{\partial}{\partial Q_{j}}\left[\sum_{j} \omega_{j} \tanh \left(\beta \hbar \omega_{j} / 2\right) Q_{j}^{2}-\alpha \sum_{j} c_{j} Q_{j}\right]=0,
$$

where $\alpha$ is a Lagrange multiplier, the following expression for the most probable values of the oscillators coordinates $Q_{j}^{0}$ is readily obtained:

$$
Q_{j}^{0}=\frac{\alpha}{2} \frac{c_{j}}{\omega_{j}} \operatorname{coth}\left(\beta \hbar \omega_{j} / 2\right) .
$$

One can see that the initial displacement of the $j$ th solvent mode $Q_{j}^{0}$ is larger in the quantum case, $\operatorname{since} \tanh \left(\beta \hbar \omega_{j} / 2\right)$ is less the corresponding classical term, $\beta \hbar \omega_{j} / 2$, for the high frequency modes. To find the value of $\alpha$ which corresponds to $\Delta \nu_{0}$ Eq. (68) is introduced into Eq. (66): 


$$
\Delta \nu_{0}=\alpha \sum_{j} \frac{c_{j}^{2}}{2 h \omega_{j}} \operatorname{coth}\left(\beta \hbar \omega_{j} / 2\right) .
$$

Using the expression for $C(t)$, Eq. (17) the following estimate for $\alpha$ is readily obtained:

$$
\alpha=\frac{h^{2} \Delta \nu_{0}}{2 \pi C(0)} .
$$

Thus, the expression for the initial displacement of the $j$ th solvent mode in this off-peak excitation can be written as

$$
Q_{j}^{0}=\frac{h^{2} \Delta \nu_{0}}{4 \pi C(0)} \frac{c_{j}}{\omega_{j}} \operatorname{coth}\left(\beta \hbar \omega_{j} / 2\right) .
$$

This expression gives the displacements of the solvent modes relative to their minima in the ground electronic state of the solute. The time-evolution, however, proceeds in the excited electronic state. The initial displacement of the $j$ th solvent mode relative to its minimum in the excited electronic state is [cf. Eqs. (2) and (A1)]:

$$
Q_{j}^{\prime 0}=\frac{c_{j}}{\omega_{j}^{2}}+Q_{j}^{0}=\frac{c_{j}}{\omega_{j}^{2}}\left[1+\frac{h \Delta \nu_{0}}{C(0)} \frac{\hbar \omega_{j} / 2}{\tanh \left(\hbar \omega_{j} \beta / 2\right)}\right] .
$$

The first term in Eq. (72) describes the displacement of the $j$ th mode when the pump pulse frequency is tuned to the maximum of the absorption spectrum. It is purely classical in the harmonic oscillator model. The second term appears when there is a detuning $\Delta \nu_{0}$. It is larger for the high frequency (and hence quantum) modes because of the tunneling [see the discussion after Eq. (68)]. The time-evolution is similar both for the quantum and classical modes and is described by the factor $\cos \omega_{j} t$ for the $j$ th mode. Therefore, the DSS can be written as

$\bar{X}(t)-\bar{X}(\infty)=\sum_{j} c_{j} Q_{j}^{\prime 0} \cos \omega_{j} t=2 \lambda \Delta(t)+h \Delta \nu_{0} \Delta_{1}(t)$,

where we have used Eqs. (17), (21), (52), and (72). Comparing Eqs. (73) and (53) one sees that they coincide. Thus, the deviation of the solvent response $S(t)$ [cf. Eq. (55)] from Eq. (20) is caused by the fact that the mean displacements $Q_{j}^{\prime 0}$ of the high frequency modes immediately after the pump pulse are different in the quantum and classical cases.

It was assumed in the previous discussion that the high frequency solvent modes do not have time to change during the excitation. In particular, $\tau_{p}$ must satisfy the condition:

$$
\frac{h}{\tau_{p}} \geq k_{B} T .
$$

(A high frequency mode is defined here as one whose frequency exceeds $k_{B} T / h$.) This condition is loosely satisfied for the pump pulse with duration $\tau_{p} \simeq 50$ fs at the room temperature. On the other hand, the pulse must not be too broad in the frequency domain, since otherwise the constraint $\Delta \nu_{0}=$ const would be meaningless. Taking into account that the spectral width is $\sqrt{\lambda / \beta} / \hbar$ one arrives at the condition given by Eq. (49) and discussed above.

\section{SUMMARY}

In the present paper the effect of the pump pulse duration on the Stokes shift time-evolution was considered. It was shown that the deviation of the solvent response from the classical expression, Eq. (20), is due to the mean initial displacements of the high frequency solvent modes being different in the quantum and classical cases. It was found, however, that usually this effect is small and that the standard description using an infinitely short excitation pulse is then applicable. However, it was shown that a deviation can be expected when the excitation pulse frequency is tuned to the far wing of the absorption band of the chromophore. The description of the transient fluorescence spectrum in which the only parameter, the central frequency of the spectrum, is used to characterize its dynamics, can omit some of the dynamical features. The other features of the spectrum such as its width and shape can contain additional information about the solvation dynamics.

\section{ACKNOWLEDGMENTS}

It is a pleasure to acknowledge the support of the National Science Foundation and the Office of Naval Research. One of us (Y.G.) would like to acknowledge the support of the James W. Glanville Postdoctoral Scholarship in Chemistry at Caltech and support in the early stages of this research of the Rueff-Wormser Fellowship.

\section{APPENDIX A: DERIVATION OF EQ. ( 24)}

To derive Eq. (24) shifted normal modes $Q_{j}^{\prime}$ can be introduced:

$$
Q_{j}^{\prime}=Q_{j}+\frac{c_{j}}{\omega_{j}^{2}} .
$$

The expression for the change $\Delta X$ in the solvation coordinate $X$ in Eqs. (3) and (5) can be rewritten in terms of $Q_{j}^{\prime}$ as

$$
\Delta X=\sum_{j} c_{j} Q_{j}^{\prime}-2 \lambda
$$

using Eq. (4) for $\lambda$. The time-evolution of a shifted normal mode in the excited electronic state with the Hamiltonian $H_{e}$ [Eq. (2)] formally coincides with a time-evolution of an unshifted harmonic oscillator and is given by

$$
Q_{j}^{\prime}(t)=Q_{j}^{\prime} \cos \omega_{j} t+\frac{P_{j}}{\omega_{j}} \sin \omega_{j} t .
$$

$\Delta X_{e}(t)$ can be written as

$$
\Delta X_{e}(t)=\sum_{j} c_{j} Q_{j}^{\prime}(t)-2 \lambda=\Delta X_{g}(t)+2 \lambda[\Delta(t)-1],
$$

where we have used Eqs. (A1)-(A3), (21) and the expression for $\Delta X_{g}(t)$,

$$
\Delta X_{g}(t)=\sum_{j} c_{j}\left[Q_{j} \cos \omega_{j} t+\frac{P_{j}}{\omega_{j}} \sin \omega_{j} t\right] .
$$




\section{APPENDIX B: THE DIRECTIONAL PROPERTIES OF THE TRANSIENT FLUORESCENCE}

If the direction of the electric field $\mathbf{n}_{0}$ in the pump pulse is not collinear with the direction of the transition dipole moment of the solute $\mathbf{n}^{\prime}$, a factor of $\mathbf{n}_{\mathbf{0}} \cdot \mathbf{n}^{\prime}$ appears in the expression for the Hamiltonian of interaction of the pump pulse with the solute [cf. Eq. (26)]. Similarly, if the direction of the electric field $\mathbf{n}$ in a particular fluorescence mode is not collinear with $\mathbf{n}^{\prime}$, a factor of $\mathbf{n} \cdot \mathbf{n}^{\prime}$ appears in the corresponding interaction Hamiltonian. The transient fluorescence intensity is obtained as a result of the second order perturbation over the interaction with the pump pulse plus the second order perturbation over the interaction with the particular fluorescence mode. ${ }^{52}$ The contribution to the intensity $I_{n^{\prime}}$ of the transient fluorescence to the particular fluorescence mode from solutes with the given orientation $\mathbf{n}^{\prime}$ of the transition dipole moment can then be written as

$$
I_{n^{\prime}}=\left(\mathbf{n}_{\mathbf{0}} \cdot \mathbf{n}^{\prime}\right)^{2}\left(\mathbf{n} \cdot \mathbf{n}^{\prime}\right)^{2} I_{0},
$$

where $I_{0}$ depends neither on $\mathbf{n}_{0}$ and $\mathbf{n}$ nor on $\mathbf{n}^{\prime}$. To obtain the orientational dependence of the transient fluorescence intensity $I$ one must average Eq. (B2) over possible orientations of a solute molecule,

$$
I=n_{0 i} n_{0 j} n_{k} n_{l} \overline{n_{i}^{\prime} n_{j}^{\prime} n_{k}^{\prime} n_{l}^{\prime}} I_{0}, \quad i, j, k, l=1,2,3,
$$

where the bar means averaging over the solute orientation and the summation over repeated indices is assumed. The tensor $\overline{n_{i}^{\prime} n_{j}^{\prime} n_{k}^{\prime} n_{l}^{\prime}}$ has to be isotropic because of the solvent isotropy. The most general form of such a tensor is

$$
\overline{n_{i}^{\prime} n_{j}^{\prime} n_{k}^{\prime} n_{l}^{\prime}}=a\left(\delta_{i, j} \delta_{k, l}+\delta_{i, k} \delta_{j, l}+\delta_{i, l} \delta_{j, k}\right) .
$$

Summing the tensor $\overline{n_{i}^{\prime} n_{j}^{\prime} n_{k}^{\prime} n_{l}^{\prime}}$ over indices $i, j$ and $k, l$ and taking into account that $n_{i}^{\prime} n_{i}^{\prime}=1$ one finds that $a=1 / 15$. As a result, the fluorescence intensity can be written as

$$
I=\frac{1}{15}\left[1+2\left(\mathbf{n} \cdot \mathbf{n}_{0}\right)\right] I_{0} .
$$

\section{APPENDIX C: DERIVATION OF EQ. ( 35)}

To derive Eq. (35) it is convenient to treat the trace in this equation as a thermal average in the ground electronic state, Eq. (6), and to use the time-ordered exponential notation, ${ }^{90}$

$$
\exp _{+}\left[-\frac{i}{\hbar} \int_{0}^{t} d t^{\prime} X_{g}\left(t^{\prime}\right)\right]=e^{i t H_{g} / \hbar} e^{-i t H_{e} / \hbar}
$$

The correlation function $R(t)$, Eq. (31), can be written in this notation as

$$
R(t)=\left\langle\exp _{+}\left[-\frac{i}{\hbar} \int_{0}^{t} d t^{\prime} X_{g}\left(t^{\prime}\right)\right]\right\rangle .
$$

Using Eq. (24) the response function $f\left(t \mid t^{\prime}, t^{\prime \prime}\right)$ defined in Eq. (34) can be written as

$$
f\left(t \mid t^{\prime}, t^{\prime \prime}\right)=f_{1}\left(t \mid t^{\prime}, t^{\prime \prime}\right)+2 \lambda\left[\Delta\left(t-t^{\prime}\right)-1\right] R\left(t^{\prime}-t^{\prime \prime}\right),
$$

where

$$
f_{1}\left(t \mid t^{\prime}, t^{\prime \prime}\right)=\left\langle X_{g}\left(t-t^{\prime}\right) \exp _{+}\left[-\frac{i}{\hbar} \int_{t^{\prime \prime}-t^{\prime}}^{0} d \tau X_{g}(\tau)\right]\right\rangle .
$$

It can be shown, using a diagrammatic technique ${ }^{90}$ for example, that for a harmonic system the function $f_{1}\left(t \mid t^{\prime}, t^{\prime \prime}\right)$ is equal to

$$
\begin{aligned}
f_{1}\left(t \mid t^{\prime}, t^{\prime \prime}\right)= & {\left[\langle X\rangle-\frac{i}{\hbar} \int_{t^{\prime \prime}-t^{\prime}}^{0} d \tau\left\langle\Delta X_{g}\left(t-t^{\prime}\right) \Delta X_{g}(\tau)\right\rangle\right] } \\
& \times\left\langle\exp _{+}\left[-\frac{i}{\hbar} \int_{t^{\prime \prime}-t^{\prime}}^{0} d \tau X_{g}(\tau)\right]\right\rangle .
\end{aligned}
$$

Substituting Eq. (17) into Eq. (C5) and integrating over $\tau$, the expression for $f_{1}\left(t \mid t^{\prime}, t^{\prime \prime}\right)$ is obtained. After the substitution into Eq. (C3) it yields Eq. (35).

${ }^{1}$ R. M. Stratt and M. Maroncelli, J. Phys. Chem. 100, 12981 (1996).

${ }^{2}$ R. Jimenez, G. R. Fleming, P. V. Kumar, and M. Maroncelli, Nature (London) 369, 471 (1994).

${ }^{3}$ M. Maroncelli, J. Mol. Liq. 57, 1 (1993).

${ }^{4}$ E. A. Carter and J. T. Hynes, J. Chem. Phys. 94, 5961 (1991).

${ }^{5}$ P. F. Barbara and W. Jarzeba, Adv. Photochem. 15, 1 (1990).

${ }^{6}$ J. D. Simon, Acc. Chem. Res. 21, 128 (1988).

${ }^{7}$ E. W. Castner, Jr., B. Bagchi, M. Maroncelli, S. P. Webb, A. J. Ruggiero, and G. R. Fleming, Ber. Bunsenges. Phys. Chem. 92, 363 (1988)

${ }^{8}$ P. J. Rossky and J. D. Simon, Nature (London) 370, 263 (1994).

${ }^{9}$ P. F. Barbara, G. C. Walker, and T. P. Smith, Science 256, 975 (1992).

${ }^{10}$ G. R. Fleming and P. G. Wolynes, Phys. Today 43, 36 (1990).

${ }^{11}$ M. Maroncelli, J. MacInnis, and G. R. Fleming, Science 243, 1674 (1989).

${ }^{12}$ S. Mukamel, Principles of Nonlinear Optical Spectroscopy (Oxford University Press, New York, 1995).

${ }^{13}$ Ultrafast Dynamics of Chemical Systems, edited by J. D. Simon (Kluwer Academic, Dordrecht, 1994).

${ }^{14}$ G. R. Fleming, Chemical Applications of Ultrafast Spectroscopy (Clarendon, Oxford, 1986).

${ }^{15}$ T.-S. Yang, P. Vöhringer, D. C. Arnett, and N. F. Scherer, J. Chem. Phys. 103, 8346 (1995).

${ }^{16}$ P. Vöhringer, D. C. Arnett, R. A. Westervelt, M. J. Feldstein, and N. F. Scherer, J. Chem. Phys. 102, 4027 (1995).

${ }^{17}$ E. T. J. Nibbering, D. A. Wiersma, and K. Duppen, Phys. Rev. Lett. 66, 2464 (1991)

${ }^{18}$ P. C. Becker, H. L. Fragnito, J.-Y. Bigot, C. H. Brito Cruz, R. L. Fork, and C. V. Shank, Phys. Rev. Lett. 63, 505 (1989).

${ }^{19}$ M. Cho, J.-Y. Yu, T. Joo, Y. Nagasawa, S. A. Passino, and G. R. Fleming, J. Phys. Chem. 100, 11944 (1996).

${ }^{20}$ T. Joo and A. C. Albrecht, Chem. Phys. 176, 233 (1993).

${ }^{21}$ J.-Y. Bigot, M. T. Portella, R. W. Schoenlein, C. J. Bardeen, A. Migus, and C. V. Shank, Phys. Rev. Lett. 66, 1138 (1991).

${ }^{22}$ A. M. Weiner, S. D. Silvestri, and E. P. Ippen, J. Opt. Soc. Am. B 2, 654 (1985).

${ }^{23}$ P. Cong, H. P. Deuel, Y. J. Yan, and J. D. Simon, J. Lumin. 60, 699 (1994).

${ }^{24}$ P. Cong, Y. J. Yan, H. P. Deuel, and J. D. Simon, J. Chem. Phys. 100, 7855 (1994)

25 P. Cong, H. P. Deuel, and J. D. Simon, Chem. Phys. Lett. 212, 367 (1993).

${ }^{26}$ H. L. Fragnito, J.-Y. Bigot, P. C. Becker, and C. V. Shank, Chem. Phys. Lett. 160, 101 (1989).

${ }^{27}$ T. Joo, Y. Jia, and G. R. Fleming, J. Chem. Phys. 102, 4063 (1995).

${ }^{28}$ M. Cho and G. R. Fleming, J. Phys. Chem. 98, 3478 (1994).

${ }^{29}$ M. S. Pshenichnikov, K. Duppen, and D. A. Wiersma, Phys. Rev. Lett. 74, 674 (1995).

${ }^{30}$ P. Vöhringer, D. C. Arnett, T.-S. Yang, and N. F. Scherer, Chem. Phys. Lett. 237, 387 (1995).

${ }^{31}$ C. J. Bardeen and C. V. Shank, Chem. Phys. Lett. 203, 535 (1993).

${ }^{32}$ P. Vöhringer and N. F. Scherer, J. Phys. Chem. 99, 2684 (1995).

${ }^{33}$ Y. G. Chang and J. E. W. Castner, J. Chem. Phys. 99, 7289 (1993).

${ }^{34}$ M. Cho, S. J. Rosenthal, N. F. Scherer, L. D. Ziegler, and G. R. Fleming, J. Chem. Phys. 96, 5033 (1992). 
${ }^{35}$ D. McMorrow, W. T. Lotshaw, and G. A. Kenney-Wallace, IEEE J. Quantum Electron. 24, 443 (1988).

${ }^{36}$ M. L. Horng, J. A. Gardecki, A. Papazyan, and M. Maroncelli, J. Phys. Chem. 99, 17311 (1995).

${ }^{37}$ S. J. Rosenthal, R. Jimenez, G. R. Fleming, P. V. Kumar, and M. Maroncelli, J. Mol. Liq. 60, 25 (1994)

${ }^{38}$ S. J. Rosenthal, X. Xie, M. Du, and G. R. Fleming, J. Chem. Phys. 95, 4715 (1991).

${ }^{39}$ W. Jarzeba, G. C. Walker, A. E. Johnson, and P. F. Barbara, Chem. Phys. 152, 57 (1991)

${ }^{40}$ M. A. Kahlow, W. Jarzeba, T. J. Kang, and P. F. Barbara, J. Chem. Phys. 90, 151 (1989)

${ }^{41}$ M. A. Kahlow, T. J. Kang, and P. F. Barbara, J. Chem. Phys. 88, 2372 (1988).

${ }^{42}$ W. Jarzeba, G. C. Walker, A. E. Johnson, M. A. Kahlow, and P. F. Barbara, J. Phys. Chem. 92, 7039 (1988).

${ }^{43}$ M. A. Kahlow, T. J. Kang, and P. F. Barbara, J. Phys. Chem. 91, 6452 (1987)

${ }^{44}$ V. Nagarajan, A. M. Brearley, T.-J. Kang, and P. F. Barbara, J. Chem. Phys. 86, 3183 (1987).

${ }^{45}$ M. Maroncelli and G. R. Fleming, J. Chem. Phys. 86, 6221 (1987).

${ }^{46}$ J. E. W. Castner, M. Maroncelli, and G. R. Fleming, J. Chem. Phys. 86, 1090 (1987)

${ }^{47}$ Y. T. Mazurenko and V. S. Udaltsov, Opt. Spectrosc. 45, 765 (1978),

${ }^{48}$ B. Bagchi, D. W. Oxtoby, and G. R. Fleming, Chem. Phys. 86, 257 (1984).

${ }^{49}$ A. A. Ovchinnikov and M. Y. Ovchinnikova, Sov. Phys. JETP 29, 688 (1969).

${ }^{50}$ A. A. Abrikosov, L. P. Gorkov, and I. E. Dzyaloshinski, Methods of Quantum Field Theory in Statistical Physics (Dover, New York, 1975).

${ }^{51}$ S. Mukamel, J. Phys. Chem. 89, 1077 (1985).

${ }^{52}$ R. F. Loring, Y. J. Yan, and S. Mukamel, J. Chem. Phys. 87, 5840 (1987).

${ }^{53}$ R. F. Loring, Y. J. Yan, and S. Mukamel, Chem. Phys. Lett. 135, 23 (1987).

${ }^{54}$ A. J. Leggett, S. Chakravarty, A. T. Dorsey, M. P. A. Fisher, A. Garg, and W. Zwerger, Rev. Mod. Phys. 59, 1 (1987).

${ }^{55}$ Mukamel pointed out that a key equation of his, Eq. (28) of Ref. 51, that is used in Raman spectra, time-dependent fluorescence, and photon echoes, and that he derived using a cumulant expansion, can also be derived using a molecular harmonic oscillator model. Similarly, the expression for the reaction rate (and reorganization energy) obtained by Ovchinnikov and Ovchinnikova (Ref. 49) was obtained later by Dogonadze and coworkers using the molecular harmonic oscillator model [see, for example, J. Ulstrup, Charge Transfer Processes in Condensed Media (Springer, Berlin, 1979), p. 113, and references therein].

${ }^{56}$ V. G. Levich, Adv. Electrochem. Electrochem. Eng. 4, 249 (1966).

${ }^{57}$ N. R. Kestner, J. Logan, and J. Jortner, J. Phys. Chem. 78, 2148 (1974).

${ }^{58}$ P. Siders and R. A. Marcus, J. Am. Chem. Soc. 103, 741 (1981).

${ }^{59}$ A. Mokhtari, A. Chebira, and J. Chesnoy, J. Opt. Soc. Am. B 7, 1551 (1990).
${ }^{60}$ R. A. Marcus, Discuss. Faraday Soc. 29, 21 (1960).

${ }^{61}$ L. D. Zusman, Chem. Phys. 49, 295 (1980).

${ }^{62}$ A. Warshel, J. Phys. Chem. 86, 2218 (1982).

${ }^{63}$ D. F. Calef and P. G. Wolynes, J. Phys. Chem. 87, 3387 (1983).

${ }^{64}$ R. A. Kuharski, J. S. Bader, D. Chandler, M. Sprik, M. L. Klein, and R. W. Impey, J. Chem. Phys. 89, 3248 (1988).

${ }^{65}$ T. Fonseca, B. M. Ladanyi, and J. T. Hynes, J. Phys. Chem. 96, 4085 (1992).

${ }^{66}$ R. Kubo, M. Toda, and N. Hashitsume, Statistical Physics II (Springer, Berlin, 1995), p. 150.

${ }^{67}$ To obtain Eq. (11) from Eq. (9) the following property of $C(t)$ is used: $\left\langle\Delta X_{g}(0) \Delta X_{g}(t)\right\rangle=\left\langle\Delta X_{g}(-t) \Delta X_{g}(0)\right\rangle=C(-t)=C^{*}(t)$.

${ }^{68}$ J. S. Bader and D. Chandler, Chem. Phys. Lett. 157, 501 (1989).

${ }^{69}$ M. Maroncelli and G. R. Fleming, J. Chem. Phys. 89, 5044 (1988).

${ }^{70}$ M. Maroncelli, J. Chem. Phys. 94, 2084 (1991).

${ }^{71}$ T. Fonseca and B. M. Ladanyi, J. Phys. Chem. 95, 2116 (1991).

${ }^{72}$ K. Ando and S. Kato, J. Chem. Phys. 95, 5966 (1991).

${ }^{73}$ J. Zhu and R. I. Cukier, J. Chem. Phys. 98, 5679 (1993).

${ }^{74}$ In experiment the frequency of the fluorescence spectrum maximum is traditionally used. See, for example, M. A. Kahlow, T. J. Kang, and P. F. Barbara, J. Chem. Phys. 88, 2372 (1988).

${ }^{75}$ Equation (17) is obtained using statistical independence of different modes, $C(t)=\Sigma_{j} c_{j}^{2}\left\langle Q_{j}(t) Q_{j}(0)\right\rangle$, and the correlation function of a harmonic oscillator coordinate, $\left\langle Q_{j}(t) Q_{j}(0)\right\rangle=\hbar \cosh \left(\beta \hbar \omega_{j} / 2-i \omega_{j} t\right) /$ $2 \omega_{j} \sinh \left(\beta \hbar \omega_{j} / 2\right)$ [cf. Eqs. (8.6)-(8.8) of Ref. 76].

${ }^{76}$ M. Lax, J. Chem. Phys. 20, 1752 (1952).

${ }^{77}$ C. F. Chapman, R. S. Fee, and M. Maroncelli, J. Phys. Chem. 99, 4811 (1995)

${ }^{78}$ Y. Lin and C. D. Jonah, in Ultrafast Dynamics of Chemical Systems, edited by J. D. Simon (Kluwer Academic, Dordrecht, 1994), pp. 137-162.

${ }^{79}$ A. J. Benigno, E. Ahmed, and M. Berg, J. Chem. Phys. 104, 7382 (1996).

${ }^{80}$ H. Sumi and R. A. Marcus, J. Chem. Phys. 84, 4272 (1986).

${ }^{81}$ R. Biswas, N. Nandi, and B. Bagchi, J. Phys. Chem. B 101, 2968 (1997).

${ }^{82}$ C.-P. Hsu, X. Song, and R. A. Marcus, J. Phys. Chem. B 101, 2546 (1997).

${ }^{83}$ J. B. Hasted, Aqueous Dielectrics (Chapman and Hall, London, 1973).

${ }^{84}$ M. N. Asfar and J. B. Hasted, Infrared Phys. 18, 835 (1978).

${ }^{85}$ J. B. Hasted, S. K. Husain, F. A. M. Frescura, and J. R. Birch, Infrared Phys. 27, 11 (1987).

${ }^{86}$ G. M. Hale and M. R. Querry, Appl. Opt. 12, 555 (1973).

${ }^{87}$ E. P. Ippen and C. V. Shank, in Ultrashort Light Pulses, edited by S. L. Shapiro (Springer, Berlin, 1977), p. 83.

${ }^{88}$ Assuming that the pump and the gate pulses have the same duration, the instrument response function is defined by the convolution of a pulse intensity function $\exp \left(-2 * t^{2} / \tau_{p}^{2}\right)$ with itself (Ref. 87) and has a FWHM= $2 \sqrt{\ln 2} \tau_{p}$.

${ }^{89}$ R. P. Feynman, Statistical Mechanics (Benjamin, Reading, 1972), p. 51.

${ }^{90}$ G. D. Mahan, Many-Particle Physics (Plenum, New York, 1990). 